\title{
INMORTALIDAD Y SINEQUISMO: HACIA UNA SEMIÓTICA DE LOS SISTEMAS RELIGIOSOS
}

\author{
Gerson Tenório dos Santos
}

\author{
Programa de Pós-Graduação em Comunicação e Semiótica \\ da PUC-SP
}

\begin{abstract}
Abbagnano (1992: 684-5) advierte que hace tiempo el problema de la inmortalidad ha dejado de ser un problema vivo de la filosofía por dos razones: la primera, porque la dirección de la ética moderna ha eliminado de la moral toda dependencia de una sanción ultramundana y la segunda, porque la dirección de la filosofía moderna ha negado la eficacia del debate acerca de la inmortalidad por considerar sin sentido extender el análisis filosófico mas allá de la esfera de la existencia o de la experiencia aprehensible. De acuerdo con Abbagnano, el interés por el tema se ha limitado a la esfera de la religión y de la apologética religiosa.

Sin embargo, esto no quiere decir que el tema no esté vivo en la filosofía o en la semiótica. El problema es que después de Kant la cuestión de la inmortalidad no ha encontrado un lugar apropiado debido a la crítica sobre la posibilidad del conocimiento noumênico. Si se considera, por ejemplo, la filosofía de las formas simbólicas de Cassirer,
\end{abstract}


la teoría del sinequismo de Peirce, los estudios sobre el origen y funcionamiento del lenguaje, naturalmente el tema de la inmortalidad estará presente. Obviamente tales estudios tienen un carácter pos-kantiano y se diferencian epistemológica y ontológicamente de aquellos emprendidos por los filósofos anteriores a Kant. Tales estudios amplían el debate sobre el tema y traen nuevas contribuciones para que se entienda, especialmente en este fin de milenio, el diálogo entre ciencia y religión.

Nuestro objetivo en este artículo es destacar, a partir de algunos enfoques de la semiótica peirceana - como el sinequismo- y de la semiótica de la cultura — como la noosfera y la semiosfera-, que los estudios semióticos pueden ser muy útiles para la aclaración de los sistemas religiosos en una época en que la idea de inmortalidad del alma, tan fundamental para la cultura humana, casi no encuentra fundamento en los estudios científicos.

\section{MITO Y LENGUAJE}

No es posible cualquier abordaje sobre el lenguaje humano $-\mathrm{y}$, por lo tanto, sobre el propio hombre- sin que consideremos su íntima relación con los fenómenos mítico-religiosos. Si hoy para nosotros, hombres modernos, la máxima de Saussure de que los signos linguíísticos son arbitrarios parece plausible, lo mismo no se puede decir cuando se consideran los albores del surgimiento del lenguaje humano.

El pensamiento mítico-mágico, primer estadio del pensamiento humano y hasta hoy presente en las más diferentes tribus y pueblos de todo el mundo, presenta algunas particularidades dignas de ser tenidas en cuenta. Cassirer (1989: 85) nos revela que las representaciones míticas de la humanidad no surgieron de un mundo ya acabado del ser, ni que son meros productos de la fantasía. En verdad, «para la conciencia primitiva representan la totalidad del ser. La concepción e interpretación míticas no son adheridas por añadidura a determinados elementos de la existencia empírica, sino que la "experiencia" primaria misma está penetrada a fondo por las figuras del mito y saturada de su atmósfera» (comillas del autor). En el pensamiento mítico lenguaje y mundo se tocan y se inter-penetran mutuamente, no habiendo lugar para la disociación entre el signo y el objeto representado. 
Para Cassirer (1989: 104), los conceptos lingüísticos y abstractos del pensamiento teórico tienden primordialmente «a liberar los contenidos dados sensual o intuitivamente del aislamiento en que parecen presentársenos espontáneamente». Al elevarlos por encima de sus límites estrechos, el pensamiento teórico los asocia a otros contenidos, compáralos entre sí, organizándolos en un orden definido dentro de un contexto amplio. En este caso, el contenido inmediato es solamente la salida para la composición de un sistema cerrado en el que no existen puntos aislados, puesto que todos los elementos del sistema están interrelacionados, refiriéndose unos a los otros, explicándose y aclarándose mutuamente. Contrariamente al pensamiento teórico, el pensamiento mítico no se coloca libremente delante del contenido de la percepción para establecer relaciones y comparaciones con otros contenidos a través de la reflexión consciente. Cuando entra directamente en contacto con el contenido de la percepción, éste es por él subyugado y aprisionado. Sólo siente y conoce la inmediata presencia sensible del objeto como si todo se concentrase en este punto. Los otros pensamientos y correlaciones, como acontece en el pensamiento teórico, no existen. En este estado de unión religiosa el yo inviste su máxima energía en este único objeto.

\section{NOOSFERA: EL HÁBITAT DE LOS SISTEMAS RELIGIOSOS}

Las interesantes y provocantes observaciones de Cassirer con respecto al lenguaje mítico-religioso destacan el carácter complejo que existe entre el mundo fenomenal del lenguaje y su contraparte ontológica o metafísica, en el sentido peirceano del término. Esta doble articulación entre el mundo de los espíritus y el mundo externo también es tema de la preocupación de Edgar Morin (1988), al intentar entender los complejos hilos que enlazan el mundo cultural del hombre con la naturaleza.

Siguiendo los pasos de Popper, Morin también admite la existencia de un tercer mundo al lado del mundo de las cosas exteriores y del mundo de las experiencias vividas. Este tercer mundo es el constituido por las cosas del espíritu, productos culturales, lenguajes, teorías. Para Morin, éste es el mundo de la noosfera, término utilizado por I. E. Vernadski, inspirado en el concepto creado por Teilhard de Chardin, para referirse al mundo racional humano. Tal mundo no es 
sólo producto de la actividad humana, sino también autónomo, con desarrollo y sistema de funcionamiento propios. Morin busca, a partir de las teorías de Pierre Auger y Jacques Monod, fundamentar la idea de que la noosfera es autónoma y análoga a la propia biosfera donde nació. Monod, por ejemplo, afirma que «La noosfera por ser inmaterial, poblada solamente por estructuras abstractas presenta estrechas analogías con la biosfera donde ha emergido. Una idea transmisible constituye un ser autónomo (en el sentido en que se puede hablar de un ser matemático) dotado por sí mismo de emergencia y de teleonomia, capaz de conservarse, de crecer, de tornarse más complejo» (Monod apud Morin, 1998: 142).

En el espacio de la noosfera, el mito y la religión, a pesar de estar íntimamente vinculados al carácter subjetivo, inter-subjetivo y transsubjetivo de su origen, poseen, debido a las características de la noosfera, también una realidad objetiva para el hombre. Es en y por la noosfera con la que el hombre se comunica con su medio exterior y sus semejantes. Así, una idea, un mito, un sistema religioso son sistemas de comunicación, o sistemas sígnicos, que de cierta manera representan y presentan al hombre la realidad exterior. Tales sistemas, cuando se presentan como un todo organizado, dotado de significados propios capaces de extender y ampliar su sistema de significación en la complejidad de lo real, aparecen para sí mismos como «reales». Lo real sólo adquiere significado a medida que es representado por el propio sistema de significación de la noosfera. Tal vez no sea por otra razón que para innumerables pueblos primitivos dioses, sueños, apariciones sobrenaturales, tengan carácter de real o sagrado en contraposición a las experiencias ordinarias de la vida cotidiana, que son consideradas profanas. En un culto religioso, las manifestaciones del sagrado no son apenas meras fantasías o tapujos ideológicos de una determinada clase social. Son manifestaciones concretas, creadoras de un mundo propio, autónomo, y poseen existencia real para todos sus cultivadores.

Sin embargo, no es sólo en ese sentido en el que las producciones de la noosfera tienen carácter real. Morin propone que la noosfera pueda ser dividida en dos categorías: 1) las entidades cosmo-bio-antropomorfas, mitos y religiones, pobladas de seres con apariencias animales o humanas (genios, espíritus) y 2) las entidades logomorfas, doctrinas, teorías, filosofías, que son sistemas de ideas.

Las propias entidades logomorfas - los sistemas de ideas - tienden a tomar la noosfera como el propio mundo. Muchas de las ideas, de las teorías, de los sistemas de valores sociales se toman a sí mismos como 
realidad última, y rechazan todo sistema de ideas que se les oponga, considerándolo como mentira. Sin embargo, esto forma parte de la propia constitución de la noosfera. Como afirma Morin, todo sistema de ideas es un sistema abierto y cerrado al mismo tiempo y posee el siguiente modelo: un núcleo (axiomas, reglas fundamentales de organización, ideas maestras), subsistemas dependientes/interdependientes y un dispositivo inmunológico de protección. Aunque todo sistema de ideas sea abierto en algun nivel, una vez que de eso dependen sus confirmaciones y verificaciones del mundo exterior y de los otros sistemas de ideas, posee sin embargo, un fuerte sistema de protección y defensa contra las agresiones externas. Como sistema de autoorganización y autodefensa su manutención depende largamente de la «fe» y de la confianza inspirada por los postulados indemostrables y principios ocultos (paradigmas) que forman su núcleo duro. Morin destaca que hay sistemas de ideas, como las teorías científicas, que tienden a ser más abiertas y sistemas de ideas, como las doctrinas, que tienden más bien al cerramiento y la rigidez. Pero es necesario subrayar, una vez más, que cualquier sistema de ideas para existir necesita tener un fundamento real para que pueda tener la adhesión de los que participan de él.

Naturalmente, como advierte Morin, hay un gran peligro en transformar la racionalidad, abierta a la coherencia con lo real, en nacionalización. La nacionalización es la tendencia idealista en subyugar la realidad, el mundo exterior al sistema de ideas. «En el momento mismo en que las tomamos por la realidad, las ideas, de manera casi alucinatoria, se tornan fantasmas que escapan a la realidad. El mediador sustituye el mediatizado (el mundo, el real). El "poder absoluto de las ideas" que, según Mauss, caracteriza la Magia, se torna el resultado idealista de la absorción de los espíritus y de lo real por la idea. La idea, que transporta consigo la esencia de lo real, se vuelve entonces más real que lo real, que domina o expulsa. Aquí gana sentido la genial intuición de Wittgenstein: "La eliminación de la magia [por la teoría] tiene... el carácter de magia"» (Morin, 1998: 171-172).

\section{SEMIOSFERA: DONDE EL RELIGIOSO GANA SIGNIFICADO}

Un abordaje diferente de los sistemas religiosos, en el campo de la semiótica, es el de los semióticos eslavos. Sistemas religiosos son, par 
excellence, sistemas culturales. De los abordajes semióticos, la única teoría formal de los fenómenos culturales es la semiótica de la cultura de los eslavos Lotman, Uspenskij, Piatigorsky, Toporov e Ivanov. Tal abordaje tiene como base el formalismo ruso, la Teoría General de los Sistemas y la cibernética. Para los eslavos, la cultura se define «como un dominio de la organización (información) en la sociedad humana, en oposición a la desorganización (entropía); esto es, una organización integrada y jerárquica de sistemas de signos» (Sebeok, 1994: 163). Por lo tanto, en este abordaje el fenómeno religioso no es estudiado como algo singular, sino formando parte de un complejo sistema de signos, que Lotman llamó Semiosfera.

Semiosfera para Iuri Lotman (1996: 22) es el continuum semiótico donde están sumergidos todos los procesos comunicativos y de producción de nuevas informaciones. Tales sistemas no están aislados. Son formaciones semióticas de diversos tipos y que se hallan en diversos niveles de organización. Lotman crea tal concepto, análogamente al concepto de biosfera construido por V. I. Vernadski, que utiliza, como vimos anteriormente, el concepto de noosfera para la esfera de las producciones humanas. Lotman insiste en aclarar que semiosfera no debe confundirse con noosfera: «La noosfera se forma cuando en este proceso [aquel de la biosfera que transforma la energía radiante del sol en energía química y física] adquiere un papel dominante la razón del hombre. Mientras que la noosfera tiene una existencia material y espacial y abarca una parte de nuestro planeta, el espacio de la semiosfera tiene un carácter abstracto» (Lotman, 1996: 22-23). Tal como no es posible la vida fuera de la biosfera, nos es posible, dice Lotman, la existencia misma de la semiosis fuera de la semiosfera. Todo acto particular de comunicación sólo tiene significación porque está inserto en este universo.

Lotman (op. cit.) destaca que existen algunos rasgos distintivos que caracterizan la semiosfera, como: 1 . carácter delimitado y 2 . irregularidad semiótica. En otro lugar, Lotman (1990: 123-130) subraya aún que la semiosfera está marcada por el binarismo, asimetría y por su heterogeneidad. El modelo del que se vale Lotman y los eslavos para la semiosfera es el de la lengua (que es binaria), llamada por ellos de sistema modelizante primario. Es sobre esta base que construye la cultura, que sería un sistema modelizante secundario. Es en este universo, por lo tanto, en el que encontramos el arte, la filosofía y la religión. Todos esos sistemas de significación se apoyan en el modelo que la propia lengua ofrece. La dualidad sagrado/profano, caracterís- 
tica estructural de todos los mitos que tanto encanta a Mircea Eliade, tiene en la semiótica de la cultura un excelente campo de análisis, pues son los aspectos estructurales y formales los que caracterizan a los sistemas religiosos, resaltados por los estudiosos de este campo de investigación.

Tenemos, sin embargo, que considerar que el continuum que caracteriza la semiosfera de Lotman tiene matrices ontológicas y epistemológicas diferentes del sistematizado por Peirce, llamado sinequismo. Lotman (1996: 21-22), por ejemplo, resalta que a pesar de que la semiótica de Peirce difiere de la linguística de Saussure, ambas poseen una característica común: parten de elementos atómicos y aislados (el signo, en el caso de Peirce) y «todo lo que sigue es considerado desde el punto de vista de la semejanza con él» (Lotman, 1996: 21). Creemos que hay aquí un equívoco por parte de Lotman. El signo en Peirce es dinámico, traspasa todo el cosmos y es la propia base de su doctrina sinequista, que es mucho más general que la semiosfera de Lotman. Mientras Lotman concibe la șemiosfera como un sistema cerrado y abstracto, el sinequismo peirceano es abierto y realista, lo que lleva a concebir los sistemas míticos-religiosos no de una manera formal y estructural, como hace Lotman, ya que toma la dualidad del signo lingüístico (en el sentido de Saussure) como matriz de la cultura.

\section{SINEQUISMO Y SISTEMAS RELIGIOSOS}

Como vimos, con Morin, el espacio de los sistemas religiosos es el de la noosfera. También vimos que a pesar de tener una naturaleza diferente (Lévi-Strauss y Morin la llaman de segunda naturaleza y el semiótico checo Ivan Bystrina, de segunda realidad), la noosfera posee, desde el punto de vista de su modelo de funcionamiento, claras analogías con el modo de funcionamiento de los sistemas biológicos. Sin embargo, a pesar del enfoque moriniano de procurar superar el idealismo y el dualismo, que tienen marcados los abordajes de los sistemas religiosos, se nota todavía que, para este pensador, naturaleza y cultura se constituyen en mundos diferentes.

También vimos que en la semiosfera de Lotman los sistemas religiosos sólo poseen sentido en la relación con otros procesos de comunicación e información, pues existe entre tales sistemas un continuum, 
una interdependencia entre parte/todo. Sin embargo, este espacio, como resalta el autor, es cerrado, abstracto y diferenciado tanto de la biosfera cuanto de la noosfera, lo que da a su teoría coloraciones idealistas. La contribución de esta teoría al estudio del fenómeno religioso se limita al carácter estructural de la comprensión del modelo binario que marca tanto la lengua como la cultura.

Difícilmente, los abordajes que se hacen sobre los sistemas culturales y religiosos escapan de estas trampas armadas por el pensamiento empírico-racionalista, dualista y pos-kantiano.

Aún hoy tendemos a ver lo que es material perteneciendo a un dominio (el de la naturaleza) y lo que es inmaterial perteneciendo a otro dominio (el del espíritu), lo que nos lleva a hablar de biosfera, por un lado, y de noosfera o semiosfera, por otro. Aún existen también aquellos que abogan solamente por la existencia de uno u otro dominio, descartando la existencia del otro.

Peirce, en su doctrina del sinequismo, se opone a las tres tendencias que, como destacamos arriba, predominan actualmente en el pensamiento humano. Así, en contraposición al materialismo, para quien la materia es todo, al idealismo, que aboga que las ideas son absolutas, y al dualismo, que divide todo en dos, Peirce defendió el sinequismo como la tendencia a considerar el todo como un continuo.

Para Peirce (CP 7.566), el sinequismo gobierna el dominio de la experiencia en todos sus elementos. Una proposición que no tenga cualquier relación con la experiencia se le priva de todo significado. Al oponerse al «ser es; el no-ser no es» de Parménides, Peirce declara que para el sinequismo tal pensamiento es incorrecto, pues ser es una cuestión de más o menos. Ninguna cuestión experiencial puede ser respondida con absoluta certeza.

Peirce asevera que el sinequismo no admite que los fenómenos físicos y psíquicos sean enteramente distintos, aunque pertenezcan a diferentes categorías de substancias o que funcionen como lados completamente diferentes de un escudo. Para él, todos los fenómenos tienen un solo carácter, a pesar de ser algunos más mentales y espontáneos y otros más materiales y regulares. Éstos presentan de manera semejante una mezcla de libertad y restricción, lo que permite a ellos ser; aún más, los hacen teleológicos o intencionales. Peirce, llevando el dialogismo al extremo, advierte que un sinequista no debe decir «Yo soy completamente yo y nada tengo que ver con usted». En primer lugar, afirma, porque todo vecino es, en una medida, usted mismo 
$\mathrm{y}$, en segundo lugar, porque todos los hombres que son semejantes a usted y están en circunstancias análogas son, en cierta medida, usted mismo.

Para Peirce, «el sinequismo niega que haya cualquier diferencia inconmensurable entre fenómenos; y justamente por eso no hay ninguna diferencia inconmensurable entre estar despierto o adormecido. Cuando se duerme, no se está tan profundamente adormecido cuanto se imagina que esté» (CP 7.573).

La doctrina del sinequismo se agrupa con la teoría de los signos y con el edificio filosófico erigido por Peirce. De esa manera puede comprenderse el universo y la lógica que lo gobierna. Como se sabe, Peirce defiende la idea de que todo el Universo está traspasado por signos, si es que no está exclusivamente compuesto por ellos, y declara que la tendencia de todo signo es el crecimiento. Así, el material del Universo para su continuidad es la acción de los signos.

No es por otra razón por la que «una vez que se tenga abrazado el principio de la continuidad ningún tipo de explicación de las cosas nos satisfacerá, excepto que ellas crecen» (CP 1.175). Esto nos remite a otro concepto fundamental de la metafísica peircena, que es el del falibilismo. Como nos dice Santaella (1999: 305), «El sinequismo, o principio de la continuidad, es la idea del falibilismo objetivado. Si el falibilismo es la teoría de que nuestro conocimiento nunca es absoluto, sino que navega en un continuum de incertidumbre e indeterminación, el principio de la continuidad dice que todas las cosas también navegan en ese continuum». En la Metafísica de Peirce «el substrato ontológico del falibilismo», como señala Ibri (1992: 52), «es, de un lado, el reconocimiento del acaso como un principio real responsable por los apartamientos del facto en relación con la ley, y, de otro, el entretejimiento entre acaso y ley configurando el evolucionismo». El falibilismo asevera que las leyes de la naturaleza no son absolutas. Siempre hay un principio de incertidumbre y acaso comandando la evolución del Universo. En el caso de que las leyes sean absolutas, éstas serían ciegas e inexplicables» y, consecuentemente, «bloquearían el camino de la investigación» (CP 1.175). Así, el conocimiento, la investigación son siempre abiertos, indeterminados, y es justamente esto lo que garantiza su crecimiento, su evolución. El carácter sinéquico, dinámico, en constante crecimiento y evolución de la realidad, es el propio fundamento del acto de cognición, pues «la realidad de las cosas consiste en forzar persistentemente sobre nuestro reconocimiento. Si una cosa no tuviese tal persistencia, sería un mero sueño. Realidad, por lo tanto, es persis- 
tencia, regularidad. En el caos original, donde no había regularidad, no había existencia. Todo era un sueño confuso. Podemos suponer que esto aconteció en un infinitamente pasado distante. Sin embargo, como las cosas se están tornando más regulares, más persistentes, están quedando menos oníricas y más reales» (CP 1.175).

Naturalmente dentro de un abordaje de la realidad como la del sinequismo no es posible concebirse la cuestión de la verdad como algo absoluto, como defienden muchos sistemas filosóficos y religiosos dogmáticos, o como algo extremamente relativista o inexistente, como quieren algunas doctrinas empiristas. «Verdad es conformidad de un representamen con su objeto» (CP 5.554). Una vez que toda ley es evolutiva, ya que su cierne está en el falibilismo, la verdad nunca puede ser absoluta. Su medida siempre estará relacionada con las condiciones de producción de la propia realidad. «Peirce hace de la falibilidad el nudo gordiano del coraje y de la energía para la verdad, sabiendo que la verdad está siempre constreñida por la realidad» (Santaella, 1999: 306).

¿Qué consecuencias tienen tales concepciones para la comprensión de los sistemas religiosos? ¿Cómo pueden contribuir la tendencia del universo al crecimiento, la verdad como correspondencia y la indeterminación para la comprensión de los fenómenos mítico-religiosos? Ante todo, es preciso tener claro que tales fenómenos, típicamente humanos, tienen un característica mental $\mathrm{y}$, por lo tanto, una realidad sujeta a ciertas reglas. Mental y real, como vimos arriba con Peirce, sólo varían en grado. Tales fenómenos también son sígnicos, como aseveramos con Cassirer, $y$, así, poseen una lógica posible para ser estudiada por la Semiótica. Aún más: tienen consecuencias concretas, prácticas para todos sus practicantes o simpatizantes, esto es, poseen una verdad constreñida por la realidad. Es, pues, imposible para un abordaje como el del sinequismo, no concebir las producciones mítico-religiosas como relacionadas - y también determinadas - por la misma evolución que permitió la emergencia del cerebro y del cuerpo humano. El propio Peirce hizo varios comentarios sobre la relación entre sinequismo e inmortalidad, uno de los pilares fundamentales de todo y cualquier sistema religioso.

\section{INMORTALIDAD Y SINEQUISMO}

Al comentar un pasaje de un himno brahmánico que dice «Yo soy el puro e infinito Self, soy el extático, eterno, manifiesto, el que todo 
atraviesa; soy el substrato de todo lo que posee nombre y forma», Peirce dice que toda comunicación de mente a mente se da por medio de la continuidad del ser. Así, todo hombre que se atribuye un papel en el drama de la creación, a medida que se pierde en aquel papel, se identifica con su Autor (CP 7.572). Aquí, Peirce, coherentemente con su doctrina del sinequismo, no ve al creador y la criatura como estancias aisladas y asimétricas en el teatro de la creación. Tal concepción tiene aún consecuencias más significativas para los estudios de los sistemas religiosos.

Peirce afirma, por ejemplo, que «el sinequismo se resiste a acreditar que cuando la muerte llega, la conciencia carnal cesa de inmediato» (CP 7.574). La manera como eso ocurre, señala, es difícil de decir, pues aquí como en otros lugares «el oráculo sinequista es enigmático» (ibidem). Para él, el sinequismo reconoce, en primer lugar, que la conciencia carnal es solamente una pequeña parte del hombre y, en segundo lugar, que existe «la conciencia social por medio de la cual un espíritu humano está incorporado en otros y que continúa viviendo y respirando mucho más tiempo de lo que suponen los observadores superficiales» (CP 7.575). Y esto no es todo, continúa: el hombre es un ser capaz de una conciencia espiritual, que lo constituye como el ser de verdades eternas y que está incorporada en el universo como un todo. Usando conceptos semejantes a los que Jung utilizaría más tarde, Peirce afirma que «esto como una idea arquetípica nunca puede fallar» (CP 7.576).

Para ilustrar la idea del sinequismo como inmortalidad, Peirce relata el siguiente caso:

Un amigo mío, como consecuencia de una fiebre, perdió completamente
su capacidad de oír. Antes de la tragedia, le gustaba mucho la música y, por
extraño que parezca, también después del acontecimiento adoraba quedar-
se próximo al piano cuando tocaba un buen músico. Entonces le dije: «A
pesar de todo usted puede oír un poco». «Absolutamente nada», respondió
él. «Pero yo puedo sentir la música por todo mi cuerpo». « ¿Caramba!»,
indagué, « ¿cómo es posible que un nuevo sentido sea desarrollado en
pocos meses?». «No es un nuevo sentido», respondió él. «Ahora que mi
audición se fue puedo reconocer que siempre poseí ese modo de conscien-
cia que anteriormente, junto con otras personas, confundía con audición»
(CP 7.577$)$.

Y concluye con el siguiente pasaje: «De modo semejante, cuando la conciencia carnal desaparece con la muerte, podremos percibir inmediatamente que siempre tuvimos una vívida conciencia espiritual que confundíamos con algo diferente» (Ibidem). 
Al término de esas consideraciones el propio Peirce destaca que a pesar del sinequismo no ser una religión, sino una filosofía puramente científica, puede desempeñar un importante papel en la conjunción de la religión con la ciencia.

\section{CONCLUSIÓN}

A pesar de haber sido poco trabajada en su relación con los sistemas religiosos, la teoría peirceana del sinequismo es extremamente poderosa para ofrecer una gran contribución a la semiótica de los sistemas religiosos, superando el idealismo en que se encuentra la mayoría de sus abordajes y el ostracismo a que fueron relegados por las ciencias duras, dado su carácter eminentemente mental y poco afecto a los métodos de las ciencias naturales. La inmortalidad interesa fundamentalmente a los sistemas religiosos porque, antes que nada, es la propia aspiración del cosmos. Y, como sabemos, todo el cosmos está sinéquicamente en evolución porque su objetivo último es permanecer.

\section{Referencias bibliográficas}

Abbagnano, N. (1992). Diccionario de filosofía. México: Fondo de Cultura Económica.

CASSIRER, Ernst (1989). Esencia y efecto del concepto de símbolo. México:

Fondo de Cultura Económica.

IBRI, I. A. (1992). Kósmos Noëtós. São Paulo: Editora Perspectiva.

- (1999). «Verdade e continuum». Hypnos (A filosofía: seu tempo, seus lugares) 5, 280-289. São Paulo: Centro de Estudos da Antiguidade GrecoRomana (CEAG) - PUC/SP.

Lotman, Iuri M. (1990). The Universe of the Mind - A Semiotic Theory of Culture. Bloomington: Indiana University Press.

- (1996). La semiosfera, I (Semiótica de la cultura y del texto). Madrid: Frónesis Cátedra, Universitat de València.

Morin, E. (1998). O método 4. As Idéias - Habitat, vida, costumes, organização. Porto Alegre: Editora Sulina.

PeIRCE, C. S. (1994). Collected Papers (CP). Cambridge: Harvard University Press, Database Intelex Co., vols. I a VIII. 
Santaella, M. L. (1999). «A semiótica filosófica de C. S. Peirce». Hypnos (A filosofía: seu tempo, seus lugares) 5, 301-307. São Paulo: Centro de Estudos da Antiguidade Greco-Romana (CEAG) - PUC/SP.

SEBEOK, T. A. (1988) «In what sense is language a "primary modeling system?»». En Henri Broms \& Rebecca Kaufmann (eds.), Semiotics of Culure: Proceedings of the 25 $5^{\text {th }}$ Symposium of the Tartu-Moscow School of Semiotics. Helsinki: Arator Inc. 